\title{
Processing and Transport of Retinoids by the Retinal Pigment Epithelium
}

\author{
DEAN BOK \\ Los Angeles
}

\begin{abstract}
Summary
Recent developments regarding our understanding of retinoid processing and transport during the visual cycle and related events are reviewed. Retinoids are bound and protected by a cohort of retinoid binding proteins, each of which is unique. The production of retinol (Vitamin A) derivatives is accomplished by a group of membrane-bound enzymes, some of which appear to be coupled in their actions.
\end{abstract}

\section{Historical Perspectives}

The photopigments of all organisms studied to date consist of a chromophore derived from Vitamin A (Retinol) $)^{1}$ covalently bound to a protein by a protonated aldimine bond (Schiff's base). ${ }^{2}$ The chromophore or prosthetic group is, in all cases, retinaldehyde (retinal) the oxidised form of retinol. Retinol and its derivatives are collectively referred to as retinoids and the proteins that covalently bind retinal are called opsins. Both the retinoids and the opsins are hydrophobic molecules, retinol being a fat-soluble vitamin, and the opsins belonging to a class of membrane components collectively known as intrinsic proteins. These are amphipathic molecules (meaning to tolerate both) whose fat-loving moieties are dissolved in the lipid bilayer of cell membranes and whose water-loving domains are exposed to the cytoplasm or cell exterior.

The photopigments of the vertebrate retina utilise either the 11-cis-retinal stereoisomer or the 11-cis-dehydroretinal form. Humans utilise 11-cis-retinal exclusively as far as we know. In 1960, Dowling published his classic work on a series of biochemical reactions involving the coordinated activity of the retinal pigment epithelium (RPE) and the photoreceptors. ${ }^{3}$ These events begin with the photobleaching of rhodopsin to form opsin and all-trans-retinal, the production of various retinol derivatives, the regeneration of 11-cis-retinal and ultimately, the regeneration of the photopigment itself. This complex series of interactions is known as the visual cycle. In Dowling's original observations, light was found to cause a cis to trans isomerisation of opsin-bound retinal following which retinal dissociated from the protein and was reduced to form all-trans-retinol. The all-trans-retinol was then observed to diffuse out of the photoreceptors, into the subretinal (retinal ventricular) space and into the retinal pigment epithelium (RPE) where it was converted to retinyl esters. Subsequently, in the dark, retinyl esters were hydrolysed and the photopigments were regenerated.

Initial studies of the visual cycle were not able to determine precisely the cellular sites in which all of the requisite steps take place, although it was assumed that many occurred

From: Department of Anatomy and Cell Biology and the Jules Stein Eye Institute, Center for the Health Sciences, University of California, Los Angeles, CA 90024.

Some of the work cited in this publication was supported by USPHS grants EY00444 and EY00331 and by a Center Grant from Retinitis Pigmentosa Fighting Blindness Inc. DB is Dolly Green Professor of Ophthalmology.

Correspondence to: Dean Bok, PhD, Jules Stein Eye Institute, University of California, Los Angeles, CA 90024. 
in the RPE. It was clear that all-trans-retinol was formed in the photoreceptors and that retinyl esters were formed in the RPE. Beyond that, specific details were lacking. Clearly there had to be a site of reisomerisation of the retinoids at some point and for the oxidation of retinol to retinal. In spite of an early, unconfirmed report that isomerisation was an event that occurs in the RPE, ${ }^{4}$ very little progress was made toward a further understanding of the multiple steps in the visual cycle for many years, although Krinsky demonstrated the presence of a retinyl ester synthetase in the $\mathrm{RPE}^{5}$ and Lion et al. were able to demonstrate that mammalian RPE, but not neurosensory retina, contains a stereospecific oxidoreductase capable of converting 11-cis-retinol to 11 -cis-retinal. ${ }^{6}$ Pepperberg and Masland showed that 11-cis-retinal oxidation could take place in the outer segments of amphibians. ${ }^{7}$

One of the major reasons why events in the visual cycle were so difficult to study was the extreme hydrophobicity of the retinoids and the difficulty that this property causes in aqueous systems. Furthermore, the retinoids are highly labile to oxidation and artifactual isomerisation and are difficult to separate. It was not until the advent of high performance liquid chromatography that these compounds could be resolved rapidly from complex mixtures in tissue homogenates. The lack of a wide variety of detergents for the solubilisation of membrane-bound enzymes was an important impediment to our understanding of visual cycle enzymes. Lacking also was an appreciation of the important role for retinoid binding proteins in the solubilisation of retinoids. The door to the world of these binding proteins was opened in the late 1960s with the discovery of the first retinoid binding protein.

\section{Retinoid Binding Proteins and Their Role in Retinoid Protection and Transport}

Kanai et al. reported the discovery of plasma retinol binding protein (RBP) in $1968 .{ }^{8}$ This protein is secreted by the liver as a complex with a larger protein named transthyretin (TTR) because of its binding of thyroxin and its indirect binding of retinol through its association with RBP. Retinoid binding proteins are required for the solubilisation and protection of retinoids because of their extreme hydrophobicity and susceptibility to oxidation. The solubility of retinol in water is about one nanomolar, yet its concentration in the blood is approximately one micromolar, a one thousand-fold difference. In 1976 Heller and Bok showed that RBP delivers all-transretinol to the RPE by interacting with membrane receptors on its basolateral plasma membrane (the side facing Bruch's membrane).$^{9}$ It arrives at this location by penetrating the endothelium of the choriocapillaris through its fenestrations. Once RBP delivers its cargo of retinol to the RPE, it loses its high affinity for TTR and the two diffuse back into the choriocapillaris. At the time that these phenomena were discovered, Chader and associates were discovering other, intracellular retinoid binding proteins in the retina ${ }^{10}$ and shortly thereafter, Saari and coworkers began the purification and characterisation of these proteins. ${ }^{11,12}$ They and others, including our laboratory, began the systematic localisation by immunocytochemistry of each of these binding proteins within retinal cell populations. A protein that binds all-transretinol intracellularly, named cellular retinol binding protein (CRBP) by Bashor et al. in their study of non-ocular tissues, ${ }^{13}$ was localised to RPE and Müller cells. ${ }^{14}$ Another binding protein, unique to the retina, was discovered by Futterman et al. to bind the 11-cis stereoisomers of retinol and retinal. ${ }^{15}$ Since this protein was found to bind 11-cisretinal initially, it was named cellular retinal binding protein (CRalBP). Like CRBP, it was found to exist in RPE and Müller cells. Finally, Adler et al. ${ }^{16}$ discovered another large, extracellular protein in the subretinal space which was shown to have retinoid binding properties by the laboratories of $\mathrm{Chader}^{17}$ and Bridges. ${ }^{18}$ This is a glycoprotein with low specificity for individual retinoids. It is synthesised and secreted by the photoreceptors ${ }^{19}$ and, by virtue of its location, is called interphotoreceptor or interstitial retinoid binding protein (IRBP). All of these binding proteins have been sequenced by molecular cloning methods and some by conventional protein chemistry as well. CRBP belongs to a gene family of proteins that bind retinal, retinoic acid and fatty acids (the latter are called fatty 
acid binding proteins) ${ }^{20} \mathrm{CRalBP}$ has a unique amino acid sequence and is found in no tissues outside the eye. ${ }^{21}$ IRBP is found in small quantities in the brain and is abundant in the pineal gland, a structure with primitive photoreceptive function. ${ }^{19}$ Interestingly, it has some sequence homology with a cellular retinal binding protein in cephalopods. ${ }^{22}$

The discovery of retinoid binding proteins and their localisations to specific cell types within the retina have helped significantly in our understanding of the division of labour that exists within the retina with respect to retinoids. Much remains to be learned, however about the function of these proteins. Aside from serving as protective depots for retinoids, more sophisticated functions remain to be elucidated. As yet, with the exception of RBP, none have been implicated in intra or extracellular receptor-mediated processes, although Saari and Bredberg have shown that CRalBP can serve in vitro as a substrate carrier for the oxidation of 11-cis-retinol to 11cis-retinal. ${ }^{23}$ More will be said later about other functions that might be expected of retinoid binding proteins once the critical issue of retinoid isomerisation has been discussed.

\section{Isomerisation of Retinoids for the Generation of Photopigments}

The purification and characterisation of enzymes that mediate the visual cycle has lagged far behind the purification and characterisation of retinoid binding proteins. This is understandable since, like rhodopsin most are probably intrinsic membrane proteins and therefore their purification and the retention of enzyme activity during this process is more difficult than with water-soluble enzymes. The slow progress in this area was particularly notable with respect to our understanding of the key enzyme in the visual cycle, the retinoid isomerase that regenerates 11-cis-retinoids from all-trans-retinol. Although none of the visual cycle enzymes have been fully purified, the cellular location of most of these enzymes has been known for some time, with the exception of the isomerase. The location of this enzyme was not known because its activity could not be detected in cell homogenates of either the neurosensory retina or RPE and cell and tissue culture methods also gave negative results. Likewise, since enzyme activity could not be studied there was considerable uncertainty as to the energy requirements and natural substrate for the enzyme.

These problems were recently resolved in a series of publications from Robert Rando's laboratory. First, it was shown by Bernstein and Rando ${ }^{24}$ that isomerisation occurs at the alcohol-oxidation state. Subsequently, in an interesting series of experiments, Bernstein et al. discovered that the 30 -year quest for isomerising activity had been thwarted by the organic solvents in which the substrates had been delivered to tissue homogenates. ${ }^{25}$ When the all-trans-retinol was solubilised by bovine serum albumin rather than ethanol, the traditional solvent, isomerising activity was readily detected and virtually all of this activity was found in the microsomal fraction of the RPE-choroid complex. Virtually none was found in the neurosensory retina.

Improved cell culture techniques have also supported the in vitro evidence that retinoid isomerase is an RPE enzyme. Initial attempts at the detection of isomerisation in cell cultures resulted only in the synthesis of alltrans-retinyl esters. ${ }^{26}$ There was a rapid loss of these esters from cultured cells due to the adverse affects of the high serum concentrations frequently used in the culture of RPE cells. The specific culprit in the serum appears to be albumin. ${ }^{27}$ Recently Das and Gouras have reported the synthesis of 11-cis-retinyl palmitate by cultured human RPE. ${ }^{27}$ We have succeeded in reproducing the entire RPE cell limb of the visual cycle in the culture dish by using highly-differentiated, long-term cultures of human RPE grown in only $1 \%$ serum. ${ }^{28,29}$ These cells retain their CRBP and CRalBP and express the receptor for RBP on the basolateral membrane. ${ }^{30}$ When they are provided with all-trans-retinol bound to IRBP, the putative binding protein in the subretinal space, the all-trans-retinol is converted to all-trans-retinyl palmitate, is isomerised and released back into the medium as 11-cisretinal. These experiments demonstrate the presence on an active-complete enzyme system for the visual cycle namely a retinyl ester synthetase, retinoid isomerase and a stereospecific retinol oxidoreductase. 


\section{Molecular Aspects of Enzymes Mediating the} Visual Cycle

Rando's laboratory has recently succeeded in making soluble both the retinoid isomerase activity and the retinyl ester synthetase activity from RPE and some of the molecular features of this complex enzyme system are now known. ${ }^{31}$ Efforts to date at separating the activities of retinyl ester synthetase and retinoid isomerase following solubilisation in a zwitterionic detergent have been unsuccessful. It has therefore been postulated that the two enzymes exist in the membranes of the endoplasmic reticulum as a complex. There is considerable experimental evidence to support the need for such an association. Investigators have long failed in their attempts to determine the energy source for the isomerisation reaction, an endergonic process that requires $4.1 \mathrm{kcal} / \mathrm{mol}$ of energy to drive the all-trans to 11-cis conformational change. The free energy of ester hydrolysis lies somewhere in the range of $5 \mathrm{kcal} / \mathrm{mol}$. Thus Rando and associates have proposed that the free energy of ester hydrolysis is used to drive the endergonic isomerisation reaction. ${ }^{31}$ Additional evidence from Rando's laboratory ${ }^{31}$ and that of Saari ${ }^{32}$ has made this proposal even more interesting. The fatty acid that is used to esterify retinol is derived from the number 1 position of phosphatidyl choline in the lipid bilayer of the endoplasmic reticulum. It appears that stearic acid is required in the number two position for optimal substrate recognition. ${ }^{32}$ Thus, ultimately, the energy source for retinoid isomerisation could be the fatty acyl bonds of the solvent in which the enzyme itself resides ${ }^{33}$ It is interesting to note that this scheme would not require a retinyl ester hydrolase since, by virtue of the coupled processes of ester hydrolysis and isomerisation, 11-cis-retinol could be produced directly from all-trans-retinyl ester. Nonetheless, Blaner et al. ${ }^{34}$ have shown that human RPE contains retinyl ester hydrolases that are specific for 11-cis and all-trans retinyl palmitate.

\section{Summary of Our Current Understanding of the Visual Cycle, Retinoid Processing and Retinoid Transport in the Retina}

Based on evidence presented above, our current understanding of the fate of a retinol molecule bound to RBP and entering the choriocapillaris of the eye is now relatively clear although the story is not complete. The scheme, as we currently understand it, is summarised in Figure 1. RBP interacts with its membrane receptor on the basolateral surface of the RPE and all-trans-retinol enters the cell where it is presumably bound to CRBP. The all-trans-retinol is then most likely delivered to membrane-bound retinyl ester synthetase. This enzyme removes a fatty acid, normally palmitic, from the number 1 position of phosphatidyl choline and transfers it to retinol to form retinyl esters which are either stored or used as a substrate for retinoid isomerase. It is proposed that retinyl ester synthetase (presumably synonymous with the lecithin retinol acyl transferase described by Saari and Bredberg $)^{32}$ exists as a complex with retinoid isomerase and that the free energy of retinyl ester hydrolysis is used to drive the energyrequiring isomerase reaction. The probable result is a direct conversion of all-trans-retinyl ester to 11-cis-retinol. A stereospecific 11-cisretinol isomerase then oxidises the 11-cis-retinol to 11-cis-retinal. While this process occurs the 11-cis isomer is probably bound to CRalBP. The 11-cis retinal, by an undefined process, is then released into the interphotoreceptor matrix where it is presumably protected by IRBP, using IRBP molecules in the extracellular matrix as 'stepping stones' as it diffuses to and into the rod outer segments for photopigment de novo biosynthesis or for photopigment regeneration following a light bleach.

When a photon bleaches a photopigment molecule (i.e. rhodopsin), 11-cis-retinal is converted to all-trans-retinal which is then reduced to all-trans-retinol, leaves the photoreceptor and presumably uses IRBP once again as 'stepping stones' along its diffusion path to the RPE. Upon entering the RPE, the all-trans-retinol is probably bound to CRBP and the processes of esterification and reisomerisation begin anew.

\section{Unresolved problems}

It is clear from the above description of events that a well-defined role for the retinoid binding proteins in the visual cycle, other than har- 


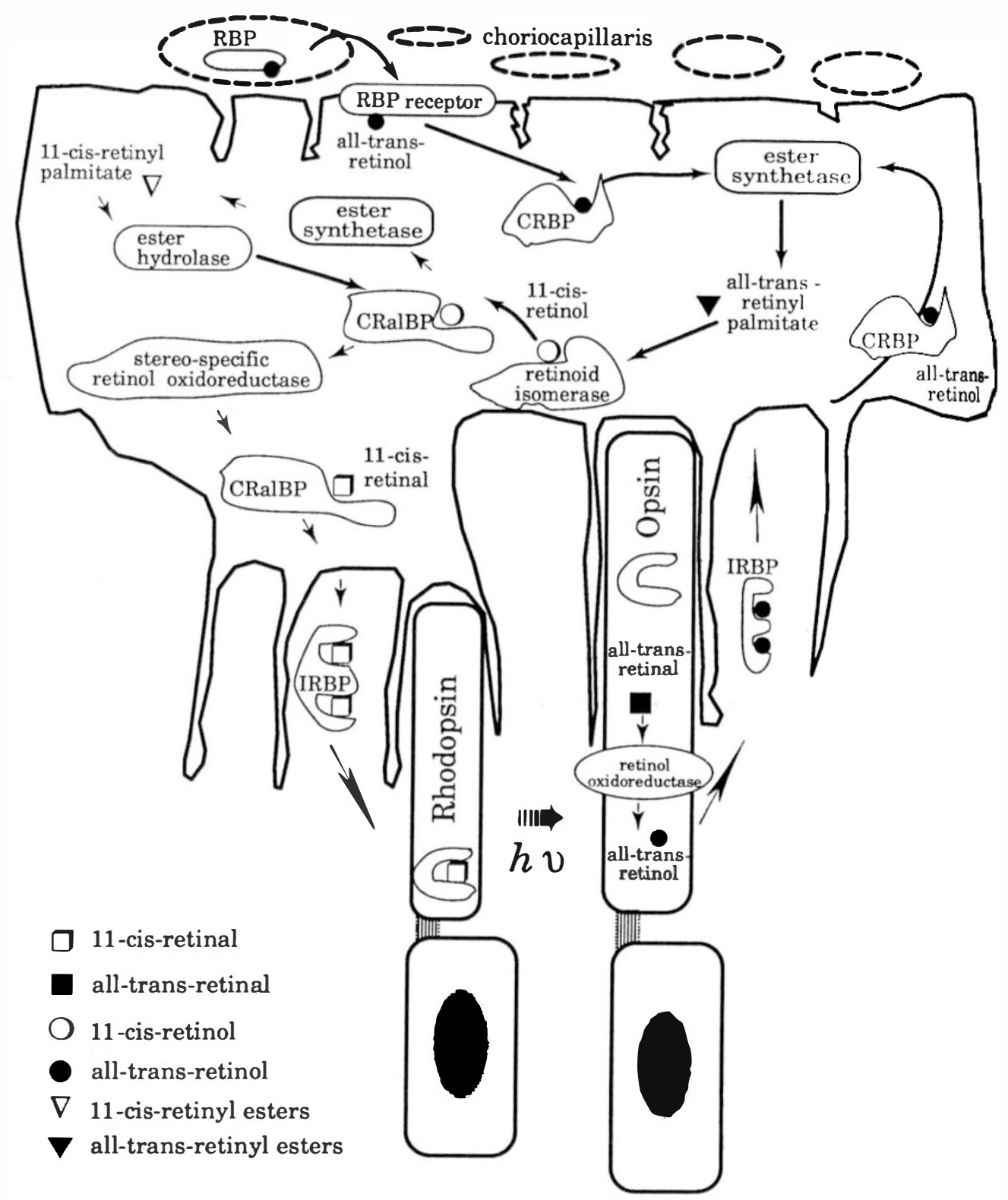

Fig. 1. Diagram showing our current concept of RPE and photoreceptor retinoids, retinoid binding proteins and enzymes thought to be involved in the visual cycle. For details, refer to the summary at the end of the text. (This figure was provided through the courtesy of John Flannery).

bouring their hydrophobic ligands, remains to be defined. Likewise, mechanisms whereby these binding proteins are relieved of their ligands needs to be resolved. Does CRalBP deliver 11-cis-retinal to the cytoplasmic side of the apical RPE plasma membrane by a receptor mediated process reminiscent of that carried out by RBP on the basolateral membrane? The role of the Müller cell in the visual cycle also remains a mystery. This cell contains the same cellular retinoid binding proteins as the RPE (CRalBP and CRBP) but it is not known whether Müller cells store retinoids. It is assumed that they do because 
CRalBP and CRBP purified from neurosensory retina contain endogenus ligands and the Müller cell is the only known repository in the neurosensory retina for these proteins. These are some of the subjects for our investigations in the future.

\section{References}

'Wald G: The molecular basis of visual excitation. Nature 1968, 219: 800-7.

${ }^{2}$ Bownds D: Sitc of attachment of retinal to opsin. Nature 1967, 216: 1178-81.

${ }^{3}$ Dowling JE: Chemistry of visual adaptation in the rat. Nature 1960, 188: 114-8.

${ }^{4}$ Hubbard R: Retinene isomerase. J Gen Physiol 1956, 39: 935-62.

${ }^{5}$ Krinsky NI: The enzymatic estcrification of vitamin A. J Biol Chem 1958, 232: 881-94.

${ }^{6}$ Lion F, Rotmans JP, Dacmen FJM, Bonting SJ: Stcreospecificity of ocular retinol dehydrogenases and the visual cyclc. Biochim Biophys Acta 1975, 384: 283-92.

${ }^{7}$ Pcpperberg DR and Masland RH: Retinal-induced sensitisation of light-adapted rabbit photoreceptors. Brain Res 1978, 151: 194-200.

${ }^{8}$ Kanai M, Raz A, Goodman DS: Retinol-bindingprotein: the transport protein for vitamin $A$ in human plasma. J Biol Chem 1968, 47: 2025-44.

${ }^{9}$ Heller J and Bok D: A specific receptor for retinol binding protein: binding of human and bovine retinol binding protein to pigment epithelial cells. Am J Ophthalmol 1976, 81: 93-7.

${ }^{10}$ Chader GJ. Wiggert B, Lai Y-L, Lec L, Fletcher RT: Interphotoreceptor retinol-binding protein: a possible role in retinoid transport to the retina. In Osborne NN, Chader GJ, (eds.) Progress in Retinal Research, New York: Pergamon Press 1982: 163-189.

${ }^{11}$ Futterman S, Saari JC, Blair S: Occurrence of a binding protein for 11-cis-retinal in retina. J Biol Chem 1977, 252: 3267-71.

${ }^{12}$ Saari JC, Futterman S, Bredberg L: Cellular retinoland retinoic acid-binding proteins of bovine retina. J Biol Chem 1978, 253: 6432-6.

${ }^{13}$ Bashor MM, Toft DO, Chytil F: In vitro binding of retinol to rat-tissue components. Proc Natl Acad Sci 1973, 70: 3483-7.

${ }^{14}$ Bok D, Ong DE, Chytil F: Immunocytochemical localisation of cellular retinol binding protein in the rat retina. Invest Ophthalmol Vis Sci 1984, 25: $1-7$.

${ }^{15}$ Saari JC, Bredberg L, Garwin GG: Identification of the endogenous retinoids associated with threc cellular retinoid-binding proteins from bovine retina and retinal pigment epithelium. J Biol Chem 1982, 257: 13329-33.

${ }^{16}$ Adler AJ and Klusznik KM: Proteins and glycoproteins of the bovine interphotoreceptor matrix: composition and fractionation. Exp Eye Res 1982, 34: 423-34.

${ }^{17}$ Lai Y-L, Wiggert B, Liu Y-P, Chader GJ: Interphotoreceptor retinol-binding proteins: possible transport vehicles between compartments of the retina. Nature 1982, 298: 848-9.

${ }^{18}$ Liou GI, Bridges CDB, Fong S-L: Vitamin A transport between retina and pigment epithelium -an interstitial protein carrying endogenous reinol (interstitial retinol binding protein). Vision Res 1982 22: $1457-68$.

${ }^{19}$ Chader GJ: Interphotoreceptor retinoid-binding protein (IRBP): A model protein for molecular biological and clinically relevant studies. Invest Ophthalmol Vis Sci 1989, 30: 7-22.

${ }^{20}$ Sundelin J, Anundi $\mathrm{H}$, Tragardh L, Eriksson U, Lind P, Ronne H, Peterson PA, Rask L: The primary structure of rat liver cellular retinol-binding protcin. Biol Chem 1985, 260: 6488-93.

${ }^{21}$ Crabb JW, Goldflam S, Harris SE, Saari JC: Cloning of the cDNAs encoding the cellular retinaldehyde-binding protein from bovine and human retina and comparison of the protein structures. $J$ Biol Chem 1988, 263: 18688-92.

${ }^{22}$ Fong S-L, Lec PG, Ozaki K, Hara R, Hara T, Bridges CDB: IRBP-like proteins in the eyes of six cephalopod species- immunochemical relationship to vertebratc interstitial retinol-binding protein (IRBP) and cephalopod retinal binding protcin. Vision Res 1988, 28: 563-74.

${ }^{23}$ Saari JC and Bredberg L: Enzymatic reduction of 11-cis-retinal bound to cellular retinal-binding protein. Biochem Biophys Acta 1982, 716: 266-72.

${ }^{24}$ Bernstcin PS and Rando RR: In vivo isomerisation of all-trans-to 11-cis-retinoids in the eyc occurs at the alconol oxidation state. Biochem 1986, 25: 6473-8.

${ }^{25}$ Bcrnstein PS, Law WC, Rando RR: Isomerisation of all-trans-retinoids to 11-cis-retinoids in vitro. Proc Natl Acad Sci 1987, 84: 1849-53.

${ }^{26}$ Flood MT, Bridges CDB, Alvarez RA, Blaner WS, Gouras P: Vitamin A utilisation in human retinal pigment cpithelial cells in vitro. Invest Ophthalmol Vis Sci 1983, 24: 1227-35.

${ }^{27}$ Das SR and Gouras P: Retionoid metabolism in cultured human retinal pigment cpithelium. Biochem J 1988, 250: 459-65.

${ }^{28}$ Flannery JG, O'Day W, Horwitz J, Bok D: The cellular compartmentalisation and role of retinoid binding proteins in the visual cycle. In Hara T (cd). Molecular Physiology of Retinal Protcins: Proceedings of the XXI Yamada Conference, Osaka: Yamada Science Foundation 1988, 261-6.

${ }^{29}$ Flannery JG, Pfeffer BA, O'Day W, Horwitz J, Bok $D$ : Isomerisation of all-trans-retinol to 11-cis-retinaldehyde by cultured human retinal pigment epithelium. Exp Eye Res 1989, (submitted).

${ }^{301}$ Pfeffer BA, Clark VM, Flannery JG, Bok D: Membrane receptors for retinol-binding protein in cultured human retinal pigment epithelium. Invest Ophthalmol Vis Sci 1986, 27: 1031-40.

${ }^{31}$ Barry RJ, Canada FJ, Rando RR: Solubilisation and partial purification of retinyl ester synthetase and retinoid isomerase from bovine ocular pigment epiuthelium. J Biol Chem 1989, 264: 9231-8.

${ }^{32}$ Saari JC and Bredberg DL: Lecithin: retinol acyltransferase in retinal pigment epithelial microsomes. J Biol Chem 1989, 264: 8638-40. 
${ }^{33}$ Deigner PS, Law WC, Canada FJ, Rando RR: Membranes as the energy source in the endergonic transformation of Vitamin A to 11-cis-retinol. Science 1989, 244: 968-71.
${ }^{34}$ Blaner WS, Das SR, Gouras P, Flood MT: Hydrolysis of 11-cis and all-trans retinyl palmitate by homogenates of human retinal epithelial cells. $J$ Biol Chem 1987, 262: 53-8. 